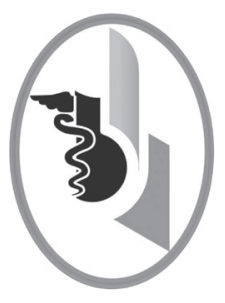

\title{
PROSTATECTOMÍA RADICAL RETROPÚBICA CON ANASTOMOSIS VESICOURETRAL CON Y SIN SUTURA. RESULTADOS POSTOPERATORIOS
}

\author{
RETROPUBIC RADICAL PROSTATECTOMY WITH CLASSIC \\ SUTURE TECHNIQUE AND SUTURELESS VESICOURETHRAL \\ ANASTOMOSIS. POSTOPERATIVE OUTCOMES
}

\section{Guarín-Cervantes Carlos Alberto ${ }^{1}$}

Ballestas-Almario Carlos

Correspondencia: cballestas24@hotmail.com

Recibido para evaluación: octubre - 15 - 2014. Aceptado para publicación: agosto - 25 - 2015.

\section{RESUMEN}

Objetivo: comparar los resultados postoperatorios de pacientes con cáncer de próstata llevados a prostatectomía radical retropúbica (PRR) con anastomosis vesicouretral con y sin sutura en Cartagena de Indias

Metodología: estudio de cohorte retrospectivo que comparó la técnica clásica de reconstrucción con sutura de la anastomosis vesicouretral durante la prostatectomía radical y la técnica de aproximación realineando la vejiga a la uretra utilizando únicamente la sonda. Se evaluaron variables preoperatorias y operatorias que incluyeron antígeno prostático, el riesgo de recurrencia del cáncer, el tiempo quirúrgico, la cantidad de sangrado, transfusiones, tiempo de sondaje y hospitalización, y durante el seguimiento la incontinencia urinaria y la estrechez uretral.

Resultados: se estudiaron 36 pacientes, a 19 (52\%) se le realizó la técnica sin sutura. Previo a la cirugía los grupos fueron homogéneos, el antígeno prostático promedio fue $9 \mathrm{ng} / \mathrm{mL}$, la mitad de los pacientes (18) tenían enfermedad de riesgo intermedio de progresión, $5(13.9 \%)$ eran de alto riesgo, 27 (75\%) tenían enfermedad limitada a la próstata en la patología, la media de tiempo quirúrgico fue 160 minutos, la mediana de la cantidad de sangrado $(600 \mathrm{cc}), 3(8.3 \%)$ requirieron transfusiones, los tiempos promedio de hospitalización fueron de tres días y de sonda 14 días, sin encontrarse diferencias estadísticamente significativas entre los dos grupos. En la evolución 14 $(47 \%)$ de los pacientes presentaron estrechez uretral, cuatro de ellos $(26.3 \%)$ del grupo sin sutura y 10 con sutura $(58.8 \%)$ p >0.05. La tasa de incontinencia urinaria e incontinencia de esfuerzos global a los tres meses fue $61 \%$ y $80 \%$ respectivamente. En igual periodo la tasa de continencia completa fue de $37 \%$ en el grupo sin sutura y $6 \%$ con sutura, de continencia parcial el $64 \%$ contra $12 \%$ respectivamente $(p<0.05)$. En 12 meses el $66.6 \%$ pacientes estaban completamente continentes, en el grupo sin sutura $16(85 \%)$ y con sutura $8(48 \%)(p<0.05)$.

Conclusión: la anastomosis vesicouretral sin sutura durante la PRR ofrece tasas de continencia temprana y completa después de un año de seguimiento superiores a la técnica con sutura. Rev.cienc.biomed. 2015;6(2):235-240.

\section{PALABRAS CLAVE}

Cáncer; Prostatectomia; Cáncer de próstata.

\footnotetext{
1 Médico. Estudiante de Postgrado. Urología. Facultad de Medicina. Universidad de Cartagena. Cartagena. Colombia.

2 Médico. Especialista en Urología. Docente Departamento Quirúrgico. Sesión de Urología. Facultad de Medicina. Universidad de Cartagena. Cartagena. Colombia
} 


\section{SUMMARY}

Objective: to compare the postoperative results of patients with prostate cancer treated with retropubic radical prostatectomy with classic suture technique and sutureless vesico-urethral anastomosis in Cartagena de Indias.

Methods: retrospective cohort study that compared the classical technique with suture reconstruction of the vesico-urethral anastomosis during radical prostatectomy and approximation technique realigning the bladder to the urethra using only a Foley catheter. We evaluated preoperative and operative variables that included PSA, the risk of recurrence, surgical time, amount of bleeding, transfusions, catheterization time and hospital stay, and follow-up urinary incontinence and urethral stricture.

Results: 36 patients participated in the study, 19 (52\%) underwent sutureless technique. Prior to surgery, the groups were similar, the average PSA was $9 \mathrm{ng} / \mathrm{mL}$, half of the patients (18) had intermediate-risk of disease progression, $5(13.9 \%)$ were high risk, $27(75 \%)$ had localized disease, the mean operative time was 160 minutes, the median amount of bleeding $(600 \mathrm{cc}), 3(8.3 \%)$ required transfusions, hospitalization 3 days and catheter time 14, non statistically significant differences were found between the two groups. In the follow-up $17(47 \%)$ patients had urethral stricture, $4(26.3 \%)$ of the sutureless and $10(58.8 \%)$ with suture group $>0.05$. The overall rate of urinary incontinence and stress incontinence at 3 months was $61 \%$ and $80 \%$ respectively. In the same period of time complete urinary continence rate was $37 \%$ in the sutureless and $6 \%$ in suture group. Partial continence was $64 \%$ versus $12 \%$ respectively $(p<0.05)$. At 12 months (66.6\%) patients were completely continent, in the sutureless $16(85 \%)$ and suture group $8(48 \%)(p<0.05)$.

Conclusion: sutureless vesicourethral anastomosis during radical retropubic prostatectomy is a simple technique with higher rates of early and complete continence after one year of follow-up compared with the suture technique. Further studies are required to corroborate the results. Rev.cienc.biomed. 2015;6(2):235-240.

\section{KEYWORDS}

Cancer; Prostatectomy; Prostate cancer.

\section{INTRODUCCIÓN}

El cáncer de próstata es la neoplasia solida más frecuente en hombres. En Colombia se estiman 8.000 nuevos casos anuales constituyendo la primera causa de incidencia de cáncer con una tasa ajustada por edad, entre 40 y $45.9 / 100.000$ hombres. Con cerca de 2.400 muertes anuales representando el $15 \%$ de las muertes por cáncer (1).

Actualmente más del $75 \%$ de los pacientes son diagnosticados con estadios tempranos, posteriormente, son tratados con prostatectomía radical o radioterapia. La prostatectomía radical retropúbica (PRR) es la cirugía estándar para el tratamiento del cáncer de próstata localizado con excelentes resultados oncológicos, sin embargo, con secuelas funcionales preocupantes. La tasa de continencia es alrededor de $80-95 \%$ al año después de la cirugía.

La anastomosis vesicouretral es un paso técnico crucial y complejo durante el procedimiento. Este afecta la continencia, las tasas de estrechez uretral, los tiempos quirúrgicos, la estancia hospitalaria y la duración del cateterismo uretral (2). La técnica clásica popularizada desde 1980 por Walsh, describe el uso de 6 puntos de sutura para reconstruir la unión vesicouretral (3). Debido a la dificultad técnica se han producido variaciones reduciendo o aumentando el número de puntos con rangos entre 2 y 8 puntos o realizando sutura continua hermética $(4,5)$.

La técnica sin sutura consiste en reconstruir la anastomosis vesicouretral únicamente afrontando y realineando la mucosa vesical a la uretra con ligera tracción con sonda Foley sin realizar puntos de sutura, esta es una técnica controversial que no se ha descrito en la literatura científica occidental, aunque se viene realizando por algunos cirujanos de forma electiva o por imposibilidad para realizar la sutura $(6,7)$. Teniendo en cuenta estos hechos, el objetivo de este estudio es comparar la incidencia de estrechez uretral, continencia urinaria, estancia hospitalaria, tiempo de duración del sondaje, tiempo quirúrgico, en una cohorte de pacientes someti- 
dos a la técnica tradicional, con sutura y sin sutura, en la anastomosis vesicouretral.

\section{MATERIALES Y MÉTODOS}

Se realizó un estudio de cohorte retrospectivo que comparó dos técnicas quirúrgicas para reconstruir la anastomosis vesicouretral. La población del estudio fueron los pacientes con cáncer de próstata a quienes se les realizó PRR en la clínica Universitaria San Juan de Dios y MediHelp Services entre julio 2010 y julio de 2012.

Los datos se obtuvieron de la descripción quirúrgica e historia clínica y fueron tabulados en la base de datos de Microsoft Excel anexando posteriormente los datos del seguimiento cada tres meses.

Las variables de medición fueron la edad, el valor del antígeno prostático previo a la cirugía, la clasificación de los pacientes según el riesgo de recurrencia, el tiempo quirúrgico, la cantidad de sangrado, transfusiones de hemoderivados durante o después del procedimiento, el tiempo en días de sondaje y de hospitalización. Durante el seguimiento incontinencia urinaria e incontinencia urinaria de esfuerzos, la última fue definida como el escape escaso de orina relacionado solamente con el esfuerzo que no requirió uso de paño. La estrechez uretral se definió como la necesidad de dilatación o uretrotomía en pacientes con síntomas urinarios obstructivos del tracto urinario inferior.

\section{Procedimiento quirúrgico}

La PRR se realizó según la técnica de Walsh por cinco cirujanos diferentes en los dos centros. En lo posible se realizó preservación del cuello vesical y de las bandeletas de acuerdo a escala de riesgo de recurrencia de cada paciente. La anastomosis vesicouretral se reconstruyó con 4-6 puntos de poliglactina y se utilizaron sondas Foley del 20-22 FR. En los pacientes sin sutura se aproximó la mucosa vesical a la uretral con el balón de la sonda 20-22 FR con leve tracción. A todos los pacientes se les dejó sonda de drenaje a retzius, recibieron antibiótico en el posoperatorio, deambulación y dieta al día siguiente de la cirugía.

\section{Análisis de datos}

Para el análisis el software se utilizó Stata (Stata 11; Statacorp; Texas, USA). Como medida de dispersión se utilizaron desviaciones estándar y rangos intercuartílicos. Con la estadistica ji-cuadrado se evaluó la significancia estadística de la asociación con la corrección de Yates para la tabla de contingencia de $2 \times 2$. Se utilizó el test exacto de Fisher cuando los valores esperados fueron menores de cinco en una celda ( $>20 \%$ de las celdas) de la tabla de contingencia de $2 \times 2$. Una $\mathrm{P}<0.05$ se consideró estadísticamente significativa.

\section{RESULTADOS}

La población del estudio fue de 36 pacientes, a 19 de ellos (52\%) se le realizó la técnica sin sutura. El tiempo de evolución registrado de cada sujeto fue de 12 meses, después de la cirugía. No se encontraron diferencias con significancia estadística en los parámetros evaluados antes de la cirugía en el grupo con sutura y sin sutura (Tabla No 1 ). La edad media de los pacientes del estudio fue de 64 años, el antígeno prostático previo a la intervención era $9 \mathrm{ng} / \mathrm{mL}$, la mitad de los pacientes (18) tenían enfermedad de riesgo intermedio de progresión, solo cinco de ellos $(13.9 \%)$ eran de alto riesgo.

En los resultados quirúrgicos no se encontraron diferencias estadísticamente significativas entre los dos grupos (Tabla No 2). La mayoría de pacientes presentaron en el resultado de patología enfermedad localizada $75 \%$, la media de tiempo quirúrgico fue mayor en el grupo de reconstrucción con sutura 160 minutos, al igual que la mediana de la cantidad de sangrado $(600 \mathrm{cc})$, tres de los pacientes $(8.3 \%)$ recibieron transfusión de glóbulos rojos, dos de estos (11.3\%) hacían parte del grupo con sutura. El tiempo de hospitalización y de sonda fue similar en los grupos.

Durante la evolución 14 de los pacientes (38.9\%) presentaron estrechez uretral. Cuatro $(26.3 \%)$ hacían parte del grupo sin sutura y diez (58.8\%) con sutura, sin obtenerse una diferencia estadísticamente significativa. La tasa de incontinencia urinaria e incontinencia de esfuerzos global a los tres 


\begin{tabular}{|c|c|c|c|c|}
\hline \multicolumn{5}{|c|}{$\begin{array}{c}\text { TABLA No } 1 . \\
\text { CARACTERÍSTICAS DE LOS PACIENTES ANTES DE LA } \\
\text { CIRUGÍA }\end{array}$} \\
\hline Variable & $\begin{array}{c}\text { Todos } \\
(n=36)\end{array}$ & $\begin{array}{l}\text { Técnica sin } \\
\text { sutura } \\
(n=19)\end{array}$ & $\begin{array}{l}\text { Técnica con } \\
\text { sutura } \\
(n=17)\end{array}$ & $\begin{array}{l}\text { Valor } \\
\text { de } p\end{array}$ \\
\hline \multicolumn{5}{|c|}{ Edad (años) } \\
\hline Mediana (RIC) & $64(61-67)$ & $64(61-68)$ & $64(62-67)$ & $0.93+$ \\
\hline \multicolumn{5}{|c|}{ PSA $(\mathrm{ng} / \mathrm{ml})$} \\
\hline Mediana (RIC) & $9(7-12)$ & $9(7.7-14)$ & $9(5.7-11.7)$ & $0.57 \dagger$ \\
\hline \multicolumn{5}{|c|}{ Riesgo } \\
\hline Bajo (\%) & $13(36.1)$ & $8(42.1)$ & $5(29.4)$ & $1.00^{*}$ \\
\hline Intermedio & $18(50.0)$ & $9(47.3)$ & $9(53.0)$ & \\
\hline Alto & $5(13.9)$ & $2(10.6)$ & $3(17.6)$ & \\
\hline
\end{tabular}

* Test exacto de Fisher, +Test de Kruskal-Wallis.

\begin{tabular}{|c|c|c|c|c|}
\hline \multicolumn{5}{|c|}{$\begin{array}{l}\text { TABLA No } 2 . \\
\text { CARACTERÍSTICAS DE LOS PACIENTES DESPUÉS DE LA } \\
\text { CIRUGÍA }\end{array}$} \\
\hline Variable & $\begin{array}{c}\text { Todos } \\
(n=36)\end{array}$ & $\begin{array}{l}\text { Técnica sin } \\
\text { sutura } \\
(\mathrm{n}=19)\end{array}$ & $\begin{array}{l}\text { Técnica con } \\
\text { sutura } \\
(n=17)\end{array}$ & $\begin{array}{l}\text { Valor } \\
\text { de } p\end{array}$ \\
\hline \multicolumn{5}{|c|}{ Patología } \\
\hline Localizado (\%) & $27(75.0)$ & $15(78.9)$ & $12(70.5)$ & $0.70 \dagger$ \\
\hline Extracapsular & $9(25.0)$ & $4(21.1)$ & $5(29.5)$ & \\
\hline \multicolumn{5}{|c|}{ Tiempo quirúrgico (min) } \\
\hline Mediana (RIC) & $\begin{array}{c}150 \\
(130-170)\end{array}$ & $\begin{array}{c}150 \\
(130-150)\end{array}$ & $\begin{array}{c}150 \\
(140-200)\end{array}$ & $0.17+$ \\
\hline \multicolumn{5}{|c|}{ Tiempo de hospitalización (días) } \\
\hline Mediana (RIC) & $3(3-4)$ & $3(3-4)$ & $3(3-4)$ & $0.51 \dagger$ \\
\hline \multicolumn{5}{|c|}{ Transfusiones (\%) } \\
\hline & $3(8.3)$ & $1(5.2)$ & $2(11.7)$ & $>0.05^{*}$ \\
\hline \multicolumn{5}{|c|}{ Cantidad de sangrado $(\mathrm{mL})$} \\
\hline Mediana (RIC) & $\begin{array}{c}425 \\
(400-750) \\
\end{array}$ & $\begin{array}{c}400 \\
(300-600) \\
\end{array}$ & $\begin{array}{c}600 \\
(400-1000) \\
\end{array}$ & $0.06+$ \\
\hline \multicolumn{5}{|c|}{ Tiempo de sondaje (días) } \\
\hline Mediana (RIC) & $14(14-15)$ & $14(14-15)$ & $14(14-15)$ & $0.61 \dagger$ \\
\hline
\end{tabular}

* Test exacto de Fisher, +Test de Kruskal-Wallis,

\begin{tabular}{|l|c|c|c|c|}
\hline \multicolumn{5}{|c|}{ TABLA No 3.} \\
\multicolumn{1}{|c|}{ CARACTERÍSTICAS DE LOS PACIENTES DURANTE EL } \\
SEGUIMIENTO \\
\hline $\begin{array}{l}\text { Variable } \\
\text { Todos } \\
(\mathrm{n}=36)\end{array}$ & $\begin{array}{c}\text { Técnica } \\
\text { sin sutura } \\
(\mathrm{n}=19)\end{array}$ & $\begin{array}{c}\text { Técnica } \\
\text { con } \\
\text { sutura } \\
(\mathrm{n}=17)\end{array}$ & $\begin{array}{c}\text { Valor } \\
\text { de } \mathrm{p}\end{array}$ \\
\hline $\begin{array}{l}\text { Incontinencia urinaria } \\
\text { a los tres meses }\end{array}$ & $\begin{array}{c}22 \\
(61.1)\end{array}$ & $7(36.84)$ & $\begin{array}{c}15 \\
(88.2)\end{array}$ & $<0.01 \dagger$ \\
\hline $\begin{array}{l}\text { Incontinencia urinaria } \\
\text { a los doce meses }\end{array}$ & $\begin{array}{c}7 \\
(19.4)\end{array}$ & $2(10.5)$ & $5(29.4)$ & $0.21 \dagger$ \\
\hline $\begin{array}{l}\text { Incontinencia urinaria de } \\
\text { esfuerzos a los tres meses }\end{array}$ & $\begin{array}{c}29 \\
(80.5)\end{array}$ & $12(63.1)$ & $16(94.1)$ & $<0.01 \dagger$ \\
\hline $\begin{array}{l}\text { Incontinencia urinaria } \\
\text { de esfuerzos a los doce } \\
\text { meses }\end{array}$ & $\begin{array}{c}12 \\
(33.3)\end{array}$ & $3(15.7)$ & $9(52.9)$ & $0.01 \dagger$ \\
\hline Estrechez uretral & $\begin{array}{c}14 \\
(38.9)\end{array}$ & $4(21.0)$ & $\begin{array}{c}10 \\
(58.8)\end{array}$ & $0.06 *$ \\
\hline
\end{tabular}

* Test exacto de Fisher, \#Test de Ji-cuadrado. meses fue de $61 \%$ en el grupo sin sutura y de $80 \%$ en el grupo con sutura respectivamente. En el mismo tiempo de seguimiento, el grupo sin sutura tuvo tasa de incontinencia urinaria de esfuerzo del $63 \%$ mientras que el $94 \%$ en el grupo con sutura fue del $94 \%$. Los resultados de la continencia urinaria están en la Tabla No 3.

\section{DISCUSIÓN}

El diagnóstico de cáncer de próstata localizado continúa creciendo. Mejorar los resultados funcionales de la cirugía radical es fundamental y se refleja en el ascendente número de publicaciones científicas para este fin. La construcción de la anastomosis vesicouretral es un factor crucial en la cirugía (8). La distribución de los grupos de este estudio fue homogénea, al igual que el comportamiento de las variables operatorias. El tiempo consumido en la construcción de la anastomosis vesicouretral con sutura, no se reflejó en una diferencia significativa del tiempo operatorio total. La ausencia de puntos que fijen la uretra a la vejiga no se tradujo en un mayor tiempo de duración con la sonda uretral.

El estadio patológico no influyó en la tasa de continencia o estrechez. El segundo objetivo clave de la prostatectomía radical después del resultado oncológico es la continencia. Los pacientes a quienes se les reconstruyó la anastomosis con puntos de sutura presentaron significativamente más incontinencia durante el seguimiento temprano. Al final del seguimiento (doce meses) de forma estadísti- 
camente significativa la tasa de continencia completa fue superior en grupo sin sutura.

Simforoosh mostró que es probable que la ausencia de puntos se refleje en un alargamiento de la uretra funcional con lo que se lograrían tasas de continencia más altas y más tempranas (7). La tasas de continencia en los pacientes sin sutura fue comparable a series actuales de estudios con la técnica con sutura. Las tasas de incontinencia en el grupo con sutura a los doce meses se encuentran por encima de $3-15 \%$ reportado por series recientes (3).

Los problemas con la anastomosis vesicouretral han sido abordados desde diferentes ángulos, por ejemplo Gallo y Mazaris mostraron similares tasas de continencia y de estrechez al reducir el número de puntos en la anastomosis $(4,9)$.

La incorporación de puntos transperineales a la anastomosis por Ingel modificando la técnica de Vest se tradujo en una tasa de continencia de $87.9 \%$ y de estrechez de $7.7 \%$ en un estudio con 91 pacientes (10).

Más recientemente Hruby desarrolló un dispositivo retráctil con seis dientes sobre un catéter uretral que unen la vejiga a la uretra mientras cicatrizan, después de probarlo en cerdos encontró resultados comparables a la técnica clásica en cuanto a continencia y estrechez, con menos reacción fibrótica en el estudio histopatológico (11).

La incidencia de estrechez uretral después de prostatectomía radical es del 0.5-32\% y en series recientes menor al 10\% (12). En los pacientes del estudio fue de $38 \%$, siendo menor en el grupo sin sutura $26 \%$ y resolviéndose en más de la mitad de los casos con dos sesiones de dilatación uretral. En el estudio de Simforoosh con reconstrucción de la anastomosis sin sutura a la tasa de estrechez fue de $13.6 \%$ (7).

La elevada tasa de incidencia de estrechez de la anastomosis en el estudio fue independiente de la presencia o ausencia de puntos de sutura. El volumen de sangrado promedio fue $400 \mathrm{cc}$, lo que se encuentra en el rango de series publicadas 350 a 1000 cc. De igual forma, los tiempos de hospitalización y de duración del sondaje (3).

En cuanto a las limitaciones se trata de un estudio retrospectivo basado en registros de historias clínicas. No fue posible la valoración endoscópica de anastomosis o tasar la continencia con instrumentos validados. Son necesarios estudios prospectivos con mayor número de pacientes para obtener resultados más concluyentes.

\section{CONCLUSIÓN}

La anastomosis vesicouretral sin sutura durante la PRR ofrece tasas de continencia temprana y completa, después de un año de seguimiento, superior a la técnica con sutura.

CONFLICTOS DE INTERESES: ninguno que declarar.

FINANCIACIÓN: recursos propios de los autores

\section{REFERENCIAS BIBLIOGRÁFICAS}

1. Piñeros M, Ferlay J, Murillo R. Cancer incidence estimates at the national and district levels in Colombia. Salud Pública Mex. 2006; 48(6): 455-65.

2. Schaeffer E, Partin A, Walsh P. Radical retropubic and perineal prostatectomy. En: CampbellWalsh Urology. 10th ed. Philadelphia: Saunders; 2011. p. 2801-2829.

3. Walsh PC. Anatomic radical prostatectomy: evolution of the surgical technique. J Urol. 1998; 160: 2418-24.

4. Mazaris E. Reducing the number of sutures for vesicourethral anastomosis in radical retropubic prostatectomy. International Braz J Urol. 2008; 35 (2): 158-63.

5. Ross PL, Carroll PR. Editorial commentre: Harpster LE, Brien J. Initial results using a running vesicourethral anastomosis following open radical retropubic prostatectomy. $\underline{\text { ] }}$ Urol. 2007;177(1):118-22.

6. Igel TC, and Wehle MJ. Vesicourethral reconstruction in radical retropubic prostatectomy: an alternative technique. J Urol. 1999; 161:844-6.

7. Simforoosh N, Javaherforooshzadeh A. Early continence after open and laparoscopic radical prostatectomy with sutureless vesicourethral alignment an alternative technique, 8 years' 
experience. Urology Journal. 2009; 6 (3): 163-9.

8. Majoros A, Bach D, Keszthelyi A, et al. Analysis of risk factors for urinary incontinence after radical prostatectomy. Urol Int. 2007; 78: 202-7.

9. Gallo L, Perdona S, Autorino R, et al. Vesicourethral anastomosis during radical retropubic prostatectomy: does the number of sutures matter? Urology. 2007; 69:547-51.

10. Igel TC, Wehle MJ. Vesicourethral reconstruction in radical retropubic prostatectomy: an alternative technique. J Urol. 1999;161: 844-6.

11. Hruby G, Weld KJ, Marruffo F, et al. Comparison of novel tissue apposing device and standard anastomotic technique for vesicourethral anastomoses. Urology. 2007;70: 190-5.

12. Park R, Martin S, Goldberg JD, Lepor H. Anastomotic strictures following prostatectomy: insights into incidence, effectiveness of intervention, effect on continence, and factors predisposing to occurrence. Urology. 2001;57: 742-6. 